\title{
Corrigendum: Estimation of the effects of radiotherapy treatment delays on tumour responses: A review
}

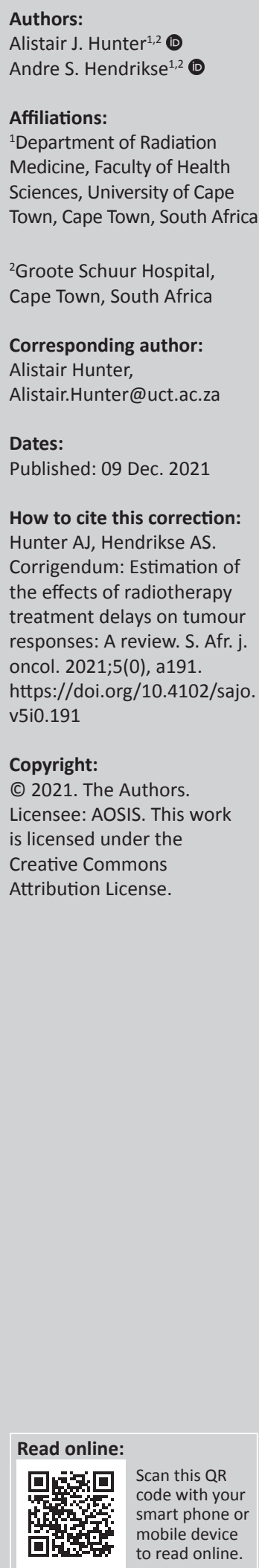

In the version of the article initially published, Hunter AJ, Hendrikse AS. Estimation of the effects of radiotherapy treatment delays on tumour responses: A review. S. Afr. j. oncol. 2020;4(0), a91. https://doi.org/10.4102/sajo.v4i0.91, the ORCID of the first author was given incorrectly. The correct ORCID should be https://orcid.org/0000-0002-6824-9342 instead of https://orcid. org/0000-0001-9282-8478 in the 'Authors' section.

This correction does not alter the study's findings of significance or overall interpretation of the study's results. The authors apologise for any inconvenience caused. 